\title{
Measurement and structure of spiral wave response functions
}

Hans Dierckx, Henri Verschelde, and Alexander V. Panfilov

Citation: Chaos 27, 093912 (2017);

View online: https://doi.org/10.1063/1.4999606

View Table of Contents: http://aip.scitation.org/toc/cha/27/9

Published by the American Institute of Physics

\section{Articles you may be interested in}

Modeling dynamics in diseased cardiac tissue: Impact of model choice

Chaos: An Interdisciplinary Journal of Nonlinear Science 27, 093909 (2017); 10.1063/1.4999605

Effects of mechanical feedback on the stability of cardiac scroll waves: A bidomain electro-mechanical simulation study

Chaos: An Interdisciplinary Journal of Nonlinear Science 27, 093905 (2017); 10.1063/1.4999465

Geometrical factors in propagation block and spiral wave initiation

Chaos: An Interdisciplinary Journal of Nonlinear Science 27, 093923 (2017); 10.1063/1.4999473

Slow $\left[\mathrm{Na}^{+}\right]$i dynamics impacts arrhythmogenesis and spiral wave reentry in cardiac myocyte ionic model

Chaos: An Interdisciplinary Journal of Nonlinear Science 27, 093907 (2017); 10.1063/1.4999475

Fast-slow asymptotic for semi-analytical ignition criteria in FitzHugh-Nagumo system

Chaos: An Interdisciplinary Journal of Nonlinear Science 27, 093916 (2017); 10.1063/1.4999472

Stochastic spontaneous calcium release events and sodium channelopathies promote ventricular arrhythmias Chaos: An Interdisciplinary Journal of Nonlinear Science 27, 093910 (2017); 10.1063/1.4999612 


\title{
Measurement and structure of spiral wave response functions
}

\author{
Hans Dierckx, Henri Verschelde, and Alexander V. Panfilov \\ Department of Physics and Astronomy, Krijgslaan 281 S9, 9000 Gent, Belgium
}

(Received 9 March 2017; accepted 27 July 2017; published online 24 August 2017)

\begin{abstract}
The rotating spiral waves that emerge in diverse natural and man-made systems typically exhibit a particle-like behaviour since their adjoint critical eigenmodes (response functions) are often seen to be localised around the spiral core. We present a simple method to numerically compute response functions for circular-core and meandering spirals by recording their drift response to many elementary perturbations. Although our method is computationally more expensive than solving the adjoint system, our technique is fully parallellisable, does not suffer from memory limitations and can be applied to experiments. For a cardiac tissue model with the linear spiral core, we find that the response functions are localised near the turning points of the trajectory. (C) 2017 Author(s). All article content, except where otherwise noted, is licensed under a Creative Commons Attribution (CC BY) license (http://creativecommons.org/licenses/by/4.0/).
\end{abstract}

[http://dx.doi.org/10.1063/1.4999606]

Spiral waves of electrical activity have been observed in the heart muscle during cardiac arrhythmias and are nowadays targeted by surgical ablation in order to cure certain heart rhythm disorders. Although mathematical models of cardiac arrhythmias may consist of a large number of coupled differential equations, it is possible to predict certain types of spiral wave drift (e.g., due to myocardial wall thickness, anisotropy, or parameter gradients) once their "response functions" are known. Here, we provide a method to experimentally measure spiral wave response functions and illustrate it for rigidly rotating and quasi-periodic (meandering) spiral waves. We test the measurement method in silico on three reactiondiffusion models. For a cardiac tissue model that supports a linear-core spiral wave, we find that the sensitivity of the system to external stimuli is concentrated near the turning points of the tip trajectory. This result suggests that methods to control the linear-core spiral waves are most effective when targeted to the position of upcoming turning points.

\section{INTRODUCTION}

Spiral waves are a prime example of self-organisation and have been observed in diverse systems, including catalytic oxidation $^{1}$ and oscillating chemical reactions. ${ }^{2}$ Moreover, they seem to be ubiquitous in biological systems, where they organise rhythmic activity from the macroscopic scale (e.g., amoeba morphogenesis ${ }^{3}$ ) down to intracellular kinetics (e.g., intracellular calcium waves ${ }^{4}$ ). Interestingly, at the tissue scale, they have been observed in neural ${ }^{5}$ and cardiac tissue, ${ }^{6-8}$ where they are thought to underlie cardiac arrhythmias.

The observation that the electrical activity in the heart during arrhythmias can be organised as spiral waves has motivated their study for decades, and in the medical community, they are known as "rotors." Recent clinical results suggest that ablating the tissue at rotor locations may cure fibrillation of the cardiac atria. ${ }^{9}$ However, if the rotors occur in the cardiac ventricles, the pumping of blood is quickly lost, and an electrical defibrillating shock needs to be administered within minutes. These examples show that a better knowledge of cardiac rotors and their dynamics may lead to better treatment or defibrillation techniques.

It was noted in some of the systems above and numerical simulations thereof that spiral waves can be remarkably stable structures. One explanation to this observation is that spiral waves are "topologically protected" structures, in the sense that when the activation phase is defined, they exhibit a phase singularity close to their rotation center, i.e., in their core region. ${ }^{8,10}$ Another possible explanation is that the tail of the spiral wave sweeps the surrounding space, resetting the medium properties to its resting values when it is passed. This way, external disturbances are strongly attenuated before they can affect the dynamics at the spiral wave core.

When the spiral wave is a dynamical attractor of the system in a two-dimensional planar geometry, its instantaneous state can be well approximated by a small set of collective coordinates, which tell how the Euclidean symmetries of the system are broken by the spiral wave solution. For a rigidly rotating spiral, we can track the spiral wave tip by computing the intersection of two isolines of variables. ${ }^{11}$ Thereby, one obtains the spiral wave tip position $(X(t), Y(t))$ in a Cartesian frame and its rotation phase $\Phi(t)$. If the spiral wave solution is quasi-periodic (meandering), the solution is only periodic modulo a Euclidean transformation. For non-resonant meander, this Euclidean transformation can be taken to be a rotation over the rotation phase $\phi$ around the centre of the meander flower. In the new frame of reference (i.e., in the quotient system of the dynamical system), the spiral solution becomes time-periodic, which can be converted to a meander phase $\Psi(t)=\Omega_{0} t+\Psi_{0}$, such that the solution becomes $2 \pi$-periodic in $\Psi$. The meander phase $\Psi(t)$ tells how far the solution has gone through the meander period. Below, we will group the collective coordinates as $X^{\mu}=(X, Y, \Phi, \Psi)$ and $\Psi$ is only included for meandering spirals.

For definiteness, we work with spiral wave solutions $\mathbf{u}(\vec{r}, t): \Omega \times \mathbb{R} \rightarrow \mathbb{R}^{N}$, i.e., the state $\mathbf{u}$ at every point of the 
domain $\Omega$ is represented as a $\mathrm{N}$-variable state vector. If one waits long enough after applying small external stimulus $\mathbf{h}$ at time $t=0$, the only persisting effect will be a net shift in the spiral's collective coordinates, i.e.

$$
\Delta X^{\mu}=\lim _{t \rightarrow \infty}\left(X^{\mu}(t)-X_{0}^{\mu}(t)\right)=W^{\mu}[\mathbf{h}(\vec{r})]
$$

for some linear functional $W^{\mu}$. When working in the continuum approximation of the medium (i.e., $\Omega \subset \mathbb{R}^{2}$, the linear functional acts as an inner product

$$
W^{\mu}[\mathbf{h}(x, y)]=\left\langle\mathbf{W}^{\mu} \mid \mathbf{h}\right\rangle
$$

with $\langle\mathbf{f} \mid \mathbf{g}\rangle=\int_{\mathbb{R}^{2}} \mathbf{f}^{H} \mathbf{g} d x d y$ and ${ }^{H}$ the Hermitian transpose. The functions $\mathbf{W}^{\mu}$ are known as the "response functions" (RFs) of the spiral wave solution. ${ }^{12}$ In essence, every point of a response function for a given state variable records the spiral wave's drift response to a perfectly localized stimulus applied to the same state variable. Using $h_{j}$ to denote the $\mathrm{j}$-th state variable component of $\mathbf{h}$, we have

$$
h_{j}=\epsilon \delta_{j}^{k} \delta\left(x-x_{0}, y-y_{0}\right) \Rightarrow \Delta X^{\mu}=\epsilon W_{k}^{\mu}\left(x_{0}, y_{0}\right) .
$$

In that sense, spiral wave RFs are similar to the impulse response in engineering or Green functions in physics. Moreover, the phase component $W^{\Psi}$ is the spatial generalisation of the phase response curve (PRC) for oscillating systems.

Knowledge of the spiral wave response functions enables predicting the spiral wave drift in the perturbative regime. Overlap integrals of spiral wave RFs thus appear in the equations of motion for three-dimensional scroll wave filaments, ${ }^{13-16}$ or in the theory of $2 \mathrm{D}$ spiral waves drifting due to a constant external field, ${ }^{17}$ surface curvature, ${ }^{18}$ or mechano-electrical feedback. ${ }^{19}$ In one spatial dimension, the translational RF of the wave front determines the velocitycurvature relation $^{20,21}$ and the shape of the RF itself can be used to shape reaction-diffusion patterns. ${ }^{22}$

If the underlying model equations are known (e.g., for a cardiac tissue model), the response functions for a (meandering) spiral can be found by linearising the system around its relative equilibrium (periodic orbit). The critical adjoint eigenfunctions to the associated linear operator are precisely the response functions; see e.g., Refs. 23-25 for details. This way, spiral wave RFs have been numerically computed, first for simple phenomenological models with few state variables $^{12,26,27}$ and later with increasing accuracy, ${ }^{28}$ up to the Beeler-Reuter cardiac tissue model. ${ }^{29}$ In all cases, the spiral wave response functions turned out to be exponentially localised in the core region, and in the view of Eq. (3), this property grants spiral waves their so-called "particle-wave dualism."12 Only for the complex Ginzburg-Landau equation, some asymptotic expressions were found. ${ }^{26}$

For meandering spiral waves, the first RFs have only been computed very recently by Marcotte and Grigoriev ${ }^{30}$ by solving the adjoint linear system. In the case of meander, the spiral wave solution and its response functions are periodic in the meander phase $\Psi$ in a slowly rotating frame. A challenge that was surmounted in the numerical computation of Ref. 30 was that the full spiral solution (with period $300 \mathrm{~ms})$ and typical discretisation time step $(0.1 \mathrm{~ms})$ do not fit into $8 \mathrm{~GB}$ of RAM memory and the solution needs to be iterated many times from the initial condition $(\Psi=0)$ over the meander period to compute the response function. For a 3-variable atrial model, the RFs as well as the first tens of leading eigenfunctions were computed in $72 \mathrm{~h}$, using graphical processing units to accelerate the time stepping in the forward problem.

In this work, we present an alternative approach to evaluate spiral wave RFs. The idea is to use Eq. (3) directly and perturb an initial condition in different simulations with a set of elementary responses and record the resulting shifts in the collective coordinates.

Section II describes the numerical methods and models used in the forward evolution, and subsequent RF reconstruction. In Sec. III, we present the RFs for a circular-core and meandering spiral in Barkley's model, and a linear core spiral in the Fenton-Karma (FK) model.

\section{METHODS}

\section{A. Theory}

We consider spiral waves as particular solutions of a non-linear dynamical system in 2 spatial dimensions $x, y$ with $N$ state variables

$$
\dot{\mathbf{u}}(x, y, t)=\hat{\mathbf{Q}}[\mathbf{u}(x, y, t)]+\mathbf{h}(x, y, t),
$$

where $\mathbf{u}(x, y, t): \mathbb{R}^{2} \times \mathbb{R}^{+} \rightarrow \mathbb{R}^{N}$. The operator $\hat{\mathbf{Q}}$ is taken to be space and time invariant (disregarding boundary conditions at a large distance). A common choice to model oscillatory and excitable media is to take a reaction-diffusion system, i.e.

$$
\hat{\mathbf{Q}}[\mathbf{u}]=\hat{\mathbf{P}}\left(\partial_{x}^{2}+\partial_{y}^{2}\right) \mathbf{u}+\mathbf{F}(\mathbf{u})
$$

A spiral wave solution solves (4) in a suitable comoving frame of reference, with drift speeds $V^{x}(\Psi, \Phi)$, $V^{y}(\Psi, \Phi)$, rotation speed $\omega_{0} \equiv V^{\theta}(\Psi)$, while its meander phase varies as $\dot{\Psi}=V^{\Psi} \equiv \Omega_{0}$. The unperturbed spiral wave solution $\mathbf{u}_{0}\left(x, y, t ; X^{\mu}(t)\right)$ thus satisfies

$$
\begin{gathered}
\mathbf{0}=\hat{\mathbf{Q}}\left[\mathbf{u}_{0}\right]+\sum_{\mu=x, y, \theta, \Psi} V^{\mu} \partial_{\mu} \mathbf{u}_{0}, \\
\dot{X}^{\mu}=V^{\mu} .
\end{gathered}
$$

The second equation describes the unperturbed spiral tip trajectory.

Ideally, one could subject the system to a stimulus (3) that is localised at a single grid point, but for small stimulus amplitude $\epsilon$, the resulting shifts $\Delta X^{\mu}$ will be below the measurement threshold in the discretised system. Therefore, we take a broader stimulus whose profile is a Gaussian kernel function, in the k-th state variable

$$
\begin{gathered}
h_{j}=\epsilon \delta(t) \delta_{j}^{k} G\left(x-x_{0}, y-y_{0}\right), \\
G(x, y)=\exp \left[-\left(x^{2}+y^{2}\right) / \sigma^{2}\right] .
\end{gathered}
$$

We choose $\epsilon$ to be equal to $0.02-0.05$ times the wave amplitude, and then choose $\sigma$ large enough to make the 
TABLE I. Parameters used in numerical methods for the different models.

\begin{tabular}{lccc}
\hline \hline Model & BA1 & BA2 & FK \\
\hline $\mathrm{dx}$ & 0.1 & 0.1 & $0.15 \mathrm{~mm}$ \\
$N_{x}$ & 400 & 400 & 600 \\
$\mathrm{dt}$ & 0.0023 & 0.0023 & $0.004 \mathrm{~ms}$ \\
$A_{u}$ & 0.05 & 0.02 & 0.05 \\
$A_{v}$ & 0.05 & 0.02 & 0.05 \\
$A_{w}$ & $\ldots$ & $\ldots$ & 0.05 \\
$\sigma_{u} / d x$ & 2 & 2 & 10 \\
$\sigma_{v} / d x$ & 2 & 2 & 10 \\
$\sigma_{w} / d x$ & $\ldots$ & $\ldots$ & 10 \\
$N_{x, s t i m}$ & 100 & 21 & 23 \\
$N_{y, s t i m}$ & 100 & 21 & 21 \\
$d t_{\text {tip }}$ & 0.1 & 0.1 & $1 \mathrm{~ms}$ \\
$t_{\text {start }}$ & 40 & 40 & $3 \mathrm{~s}$ \\
$t_{\text {end }}$ & 80 & 80 & $6 \mathrm{~s}$ \\
$\lambda$ & 10 & 500 & 10 \\
\hline \hline
\end{tabular}

coordinate shifts measurable in the system. Typically, $\sigma$ was between 2 and 10 times the simulation grid resolution, see Table I. We are also free to choose the locations of where the external stimuli are centered, and took them on a rectangular grid of spacing $\sigma$ and size $N_{x, \text { stim }} \times N_{y \text {,stim }}$ around the position of the spiral wave tip at the time of stimulus delivery. The instantaneous delivery of a stimulus, i.e., $\delta(t)$ was implemented by adding the stimulus at one time step in the simulation.

As a result, when the perturbation amplitude is large enough to dominate discretisation effects of the lattice, the shifts $\Delta^{\mu}$ will be the spatial convolution of the response function with the Gaussian kernel

$$
\Delta X^{\mu}=\epsilon \iint_{\mathbb{R}^{2}} G\left(x-x_{0}, y-y_{0}\right) W_{k}^{\mu}\left(x_{0}, y_{0}\right) d x_{0} d y_{0} .
$$

Here, $k$ is the index to the state variable for which the RF is currently probed. However, if the kernel $G$ (in simulations) or an estimate to it (in experiments) is known, one can perform a deconvolution to the measured shifts in order to reconstruct the $\mathrm{RF}$.

To allow the possibility of an unstructured grid of measurement points, we deconvolve using Tikhonov regularisation $^{31}$ rather than a Fourier-based method. Subject the system in $M$ experiments to $M$ perturbations centered at the positions $\left(x_{0}^{i}, y_{0}^{i}\right)$ and record the shifts $b_{i}=\left(\Delta X^{\mu}\right)_{i} / \epsilon$. We zero-padded the grid of responses $b_{i}$ with 5 rows in $x$ and $y$ directions.

When reconstructing the RFs in $N$ points $\left(x_{r}^{j}, y_{r}^{j}\right)$, this leads to a $M \times N$ linear system

$$
\mathbf{A w}=\mathbf{b}
$$

with $A_{i j}=G\left(x_{0}^{i}-x_{r}^{j}, y_{0}^{i}-y_{r}^{j}\right)$. Here, we choose the reconstruction grid equal to the stimulation grid. We make A sparse by rounding an element to zero if its value lies below 0.001. As a regularizing term, we take the 5-point Laplacian stencil of the set of reconstruction points, denoted by a matrix $\boldsymbol{\Gamma}$. Thereafter, we find the response function using

$$
\mathbf{w} \approx\left(\mathbf{A}^{T} \mathbf{A}+\lambda \boldsymbol{\Gamma}^{T} \boldsymbol{\Gamma}\right)^{-1} \mathbf{A}^{T} \mathbf{b} .
$$

We manually choose the lowest possible $\lambda$ which still suppresses noise outside the spiral core region, see values in Table I. The inversion is performed by the mldivide command in matlab ${ }^{32}$ using Cholesky factorization.

\section{B. Reaction-diffusion models}

For definiteness, we illustrate the method for spiral waves in a reaction-diffusion finite-difference model. It can also be applied in other systems supporting spiral waves (or, more general, relative periodic orbits), as long as Euclidean symmetry of the system is well enough preserved. Factors that may break this symmetry are the underlying discrete nature of the medium (e.g., biological cells or a computational grid), spatially varying anisotropy, and inhomogeneities in an experimental preparation or tissue.

In this work, we illustrate our methods using numerical simulations only. First, we use a reaction-diffusion model with Barkley's kinetics, ${ }^{33}$ i.e., $\mathbf{u}=[u v]^{T}, \hat{\mathbf{P}}=\operatorname{diag}(1,0)$ and

$$
\mathbf{F}(\mathbf{u})=\left(\begin{array}{c}
c^{-1} u(1-u)\left(u-\frac{v+b}{a}\right) \\
u-v
\end{array}\right) .
$$

We will take the following parameters sets:

$$
\begin{aligned}
& \text { BA1 }:\{a=1.3, b=0.19, c=0.025\}, \\
& \text { BA2 : }\{a=0.58, b=0.05, c=0.02\},
\end{aligned}
$$

which, respectively, produce a circular-core spiral wave and a meander spiral wave with flower-like tip trajectory, see Fig. 1. We found the tip position every 0.1 time units as the intersection of the isolines $u=0.5$ and $v=0.5 a-b$.

Secondly, we will determine the RFs for a cardiac tissue model with a star-like tip trajectory, which is sometimes called a "linear core." Hereto, we take the three variable Fenton-Karma (FK) model with guinea pig (GP) parameters. ${ }^{11}$ It has $\mathbf{u}=[u v w]^{T}$ and $\hat{\mathbf{P}}=\operatorname{diag}(0.1,0,0) \mathrm{mm}^{2} / \mathrm{ms}$. While $u$ is the normalized transmembrane potential, $v$ and $w$ are fast and slow recovery variables. The details of the phenomenological electrical currents in the model can be found in Ref. 11. The model produces a spiral wave with meander period $75 \mathrm{~ms}$, as shown in Fig. 1(e). We determined the tip position as the intersection of the isoline $u=0.5$ with the same curve $1 \mathrm{~ms}$ before.

The reaction-diffusion models were numerically integrated with the explicit Euler scheme one a Cartesian grid with Neumann boundary conditions, see Table I.

\section{Measurement of the shifts in collective coordinates}

From Eq. (1), the response function values can be inferred from shifts in the collective coordinates, i.e., the center, rotation angle, and time phase of the meander tip trajectories. It is thus necessary to determine accurately those shifts, at a resolution that is finer than the lattice size in simulations.

Let us first treat the circular core case, see Fig. 2(a). After applying a perturbation to a spiral wave solution at time $t=0$, we let the system evolve for several periods, and during a time interval $\left[t_{\text {start }}, t_{\text {end }}\right]$, we sample the tip trajectory 

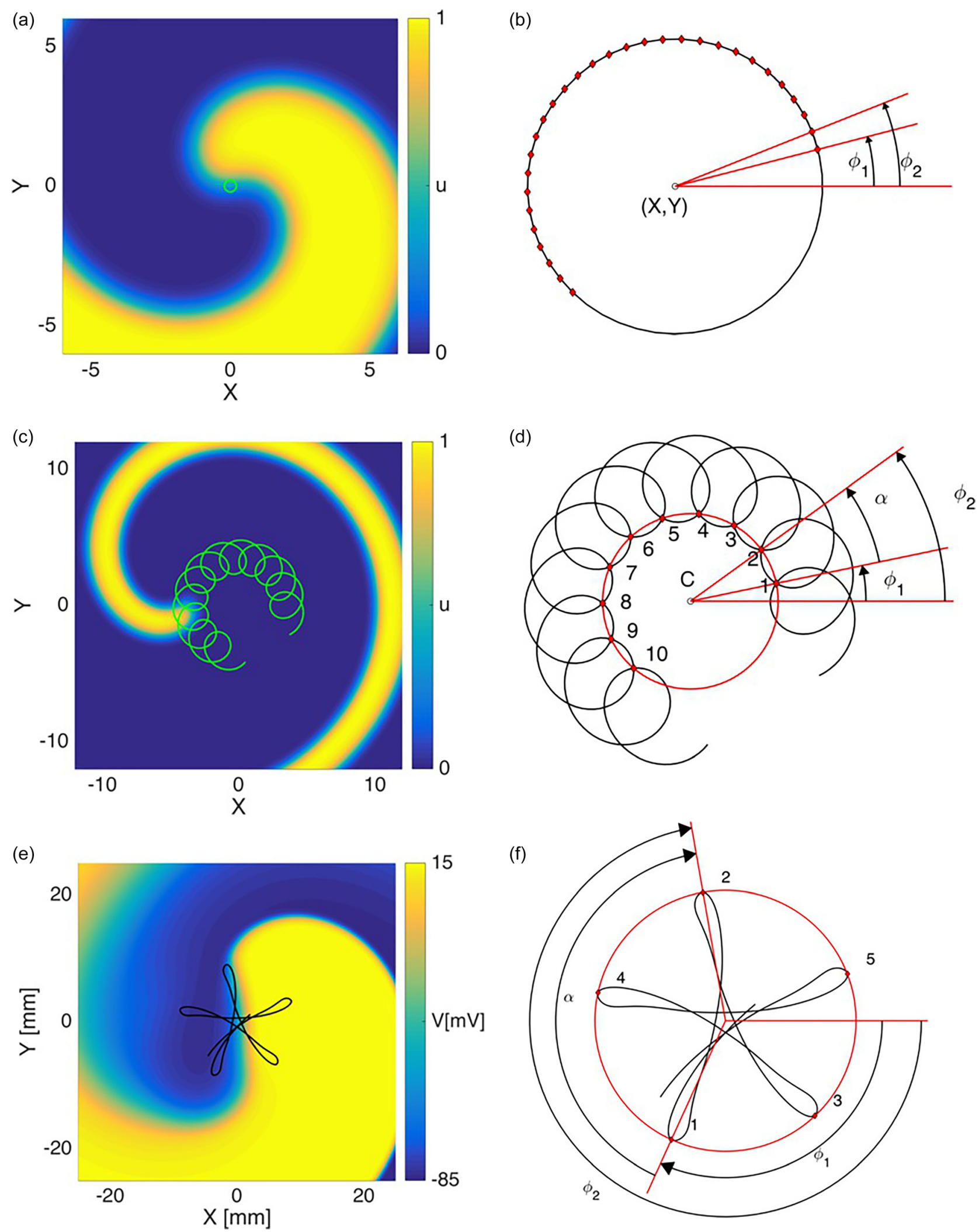

FIG. 1. Spiral waves and tip trajectories for the three reaction-diffusion models used, in Barkley's model with circular core [BA1, panel (a)] and meandering core [BA2, panel (c)] and the FK-GP model [FK, panel (e)]. Panels (b), (d), and (f) show how the collective coordinates $X, Y, \Phi, \Psi$ were fitted using fiducial points (red dots) within a meander or rotation cycle.

every $d t_{\text {tip. }}$. The $i$-th measurement is taken at time $t_{i} \in\left[t_{\text {start }}, t_{\text {end }}\right]$, yielding corresponding tip positions $\left(x_{i}, y_{i}\right)$. These points will lie approximately on a circle (see Fig. 2 left), whose center $(X, Y)$ and radius $r$ can be found by linear regression. ${ }^{34}$ Thereafter, the instantaneous rotation phase $\Phi_{i}$ can be found as the polar angle corresponding to $\left(x_{i}, y_{i}\right)$. Finally, we perform a least squares linear regression $\Phi_{i}$ $=\omega_{0}\left(t_{i}-t_{r e f}\right)+\Phi_{0}$ to obtain $\omega_{0}$ and the absolute rotation phase $\Phi_{0}$. We take $t_{\text {ref }}=\left(t_{\text {start }}+t_{\text {end }}\right) / 2$ to obtain a more robust estimate of $\Phi_{0}$. 


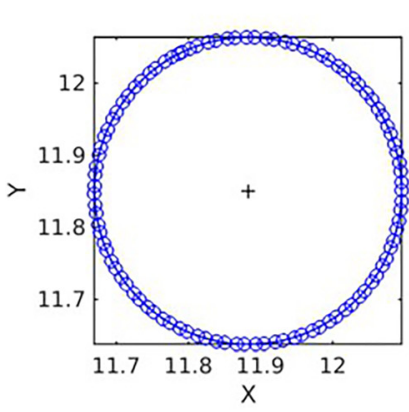

(a)
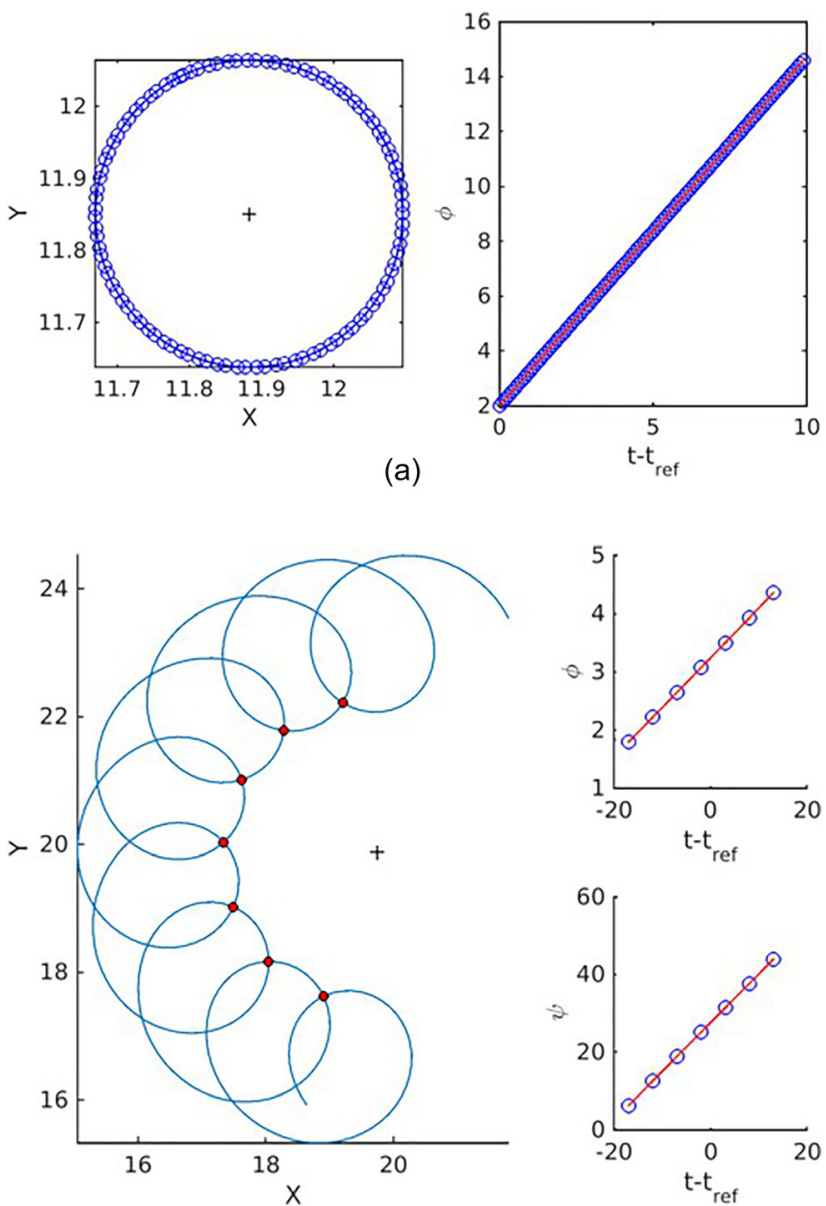

(b)

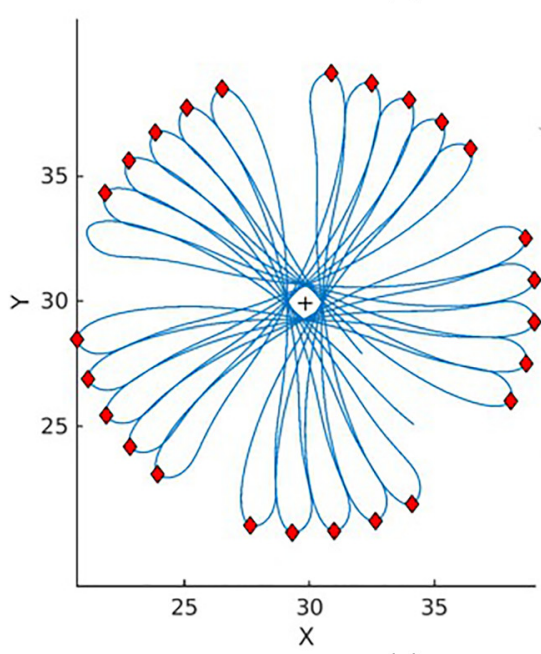

(c)
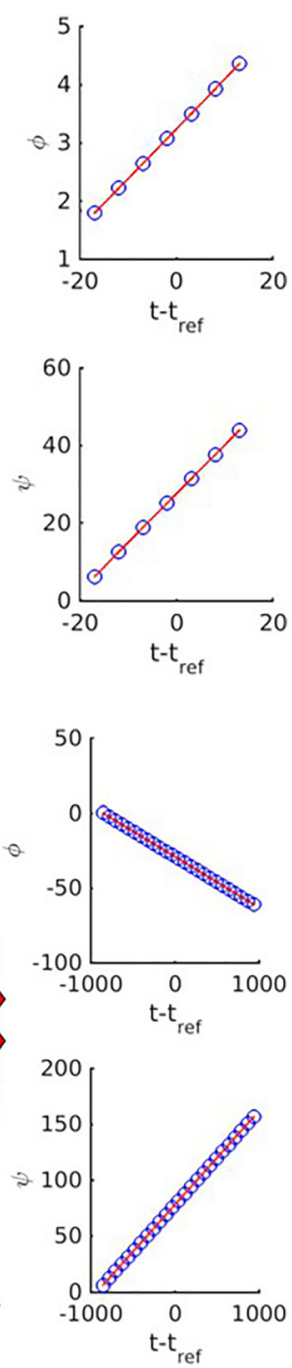

FIG. 2. Measurement of collective coordinates in the BA1 model (a), BA2 model (b), and FK model (c). For the circular-core case [panel (a)], points on the tip trajectory itself lie on a circle from which the rotation phase can be regressed. For the meander cases (b) and (c), a circle is fitted to the fiducial points (red) in the tip trajectory in order to find the position of the meander centre $(X, Y)$ (leftmost panels, indicated by + ). The polar angles $\Phi_{i}$ of the fiducial points with respect to $(X, Y)$ are regressed vs. time (top right panels) to find $\Phi_{0}$ and $\omega_{0}$. For meandering spiral waves (BA2, FK), the times at which the fiducial points are visited are used to compute the absolute meander phase $\Psi_{0}$ and meander period $T_{0}=2 \pi / \Omega_{0}$ (bottom right panels).

The case of meander requires more fine-tuning to the given tip trajectory, as shown in Figs. 2(c) and 2(d). For the BA2 model with the chosen, we first list all self-intersections of the tip trajectory. From this list, we remove self- intersections for which the time between the first and second visit is longer than 10 time units. We exclude longer time intervals, since the tip trajectory shown in Fig. 2(b) will reach again the same region after the completion of the meander flower. Such intersection points are not useful to determine the meander phase.

Since the motion is quasi-periodic, the time difference between subsequent intersection points will repeat itself after $N_{\text {rep }}$ points, with $N_{\text {rep }}$ an integer that is small if one is far from the onset of meander. To find $N_{\text {rep }}$, we looped over the possible number of classes $N_{\text {rep }}$ between 1 and 10, and computed the standard deviation at times between occurrence of a point, and found that it nearly vanished for $N_{\text {rep }}=6$ classes of intersection points. In Fig. 2(b), it can be seen that there are 3 classes of intersection points when looking at the trajectory as a whole, but they are all visited twice, and we find $N_{r e p}=6$. As each class of points lies on a circle, we estimate the centre and radius of those circles and then choose the class with smallest radius to be the set of fiducial points to determine the collective coordinates, see Figs. 1(b) and 2(b). On this circle of fiducial points, we repeat the procedure for circular-core spiral waves to find $X, Y, \Phi_{0}$ and $\omega_{0}$. At the j-th fiducial point, we also take $\Psi_{j}=$ $j 2 \pi$ and then fit $\Psi_{j}=\Omega_{0}\left(t-t_{r e f}\right)+\Psi_{0}$ to determine $\Omega_{0}$ and $\Psi_{0}$.

Thirdly, we consider the linear-core example with FK kinetics, as shown in Fig. 2(c). As the fiducial points, we will take the turning points of the trajectory, which also lie on a circle if the meander pattern is quasi-periodic. First, we smooth the tip trajectory between $t_{1}$ and $t_{2}$ using a Gaussian window of size 25. Then, we take the average of the lists $x_{i}, y_{i}$ as the first estimate of $X, Y$. Next, we determine the points where the distance to $(X, Y)$ reaches a maximum and fit a circle through them; the centre of this circle is an update estimate of $(\mathrm{X}, \mathrm{Y})$. After repeating this iterative process twice, $(X, Y)$ remained constant and were taken as the meander centre position. Thereafter, the turning points were taken as fiducial points and the procedure for flower-like meander described above was used to determine $\Phi_{0}, \Psi_{0}, \omega_{0}$, and $\Omega_{0}$.

\section{RESULTS}

\section{A. Circular-core spiral with Barkley kinetics}

For the BA1 model, we computed the three RFs using (12) and compare them to the results of solving the adjoint method in Fig. 3. For the latter, we used the publicly available software dxspiral, ${ }^{28,35}$ which was developed to compute the RFs for reaction-diffusion models that support rigidly rotating spiral waves. From Fig. 3, it can be noted that the final result using our method closely matches the result obtained using dxspiral in amplitude and shape of the profiles. For the RF measurement, we sampled the Gaussian center every two grid points of the computation grid, i.e., with spatial resolution 0.2 , to obtain the RF in a set of $101 \times 101$ points. As every forward run of 80 time units took $22 \mathrm{~s}$ on our system $(\mathrm{c}++$ program using MPI domain splitting on 16 CPUs), the total computation time amounted to $10.3 \mathrm{~h}$ per variable, which is two orders of magnitude slower 

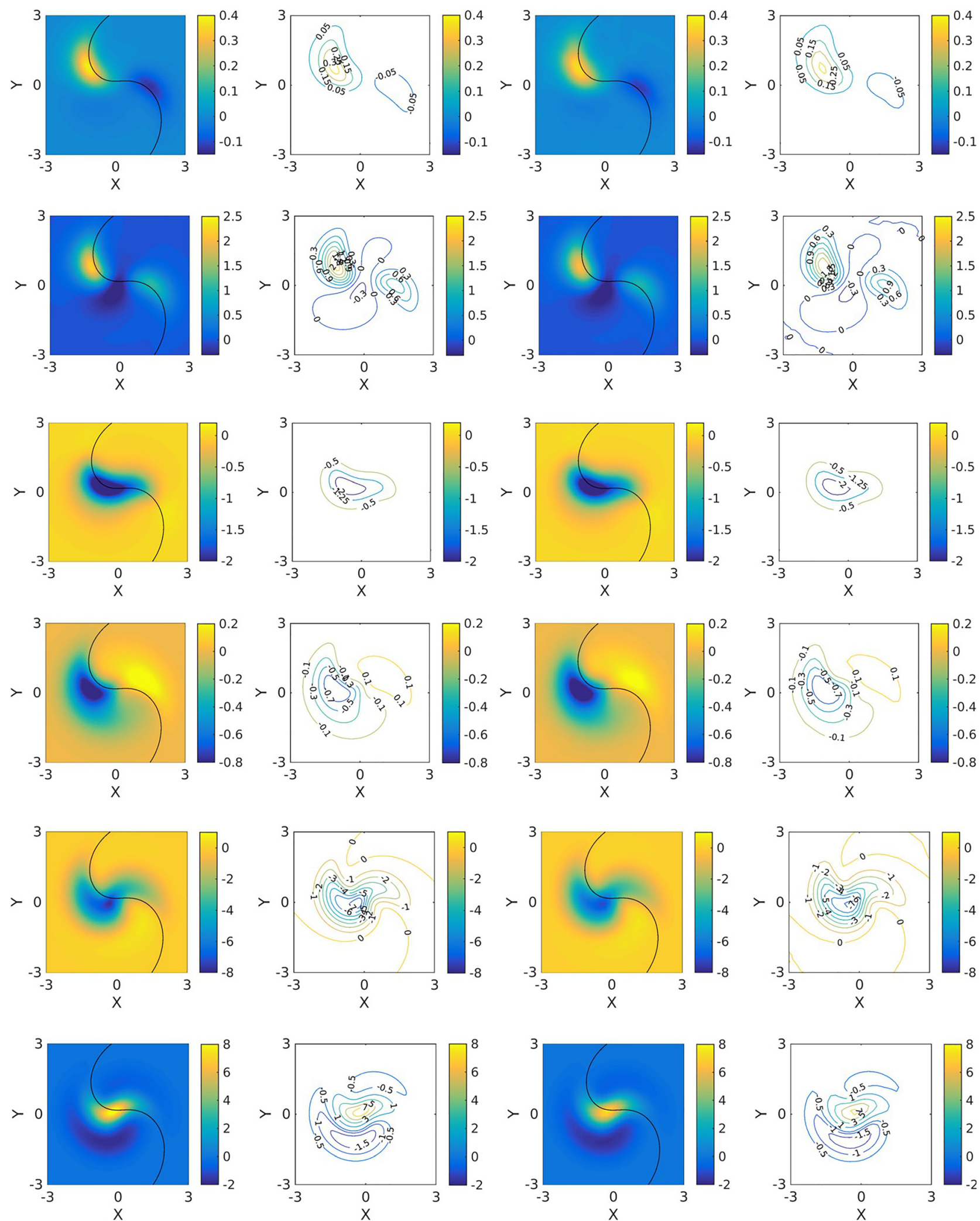

FIG. 3. Comparison of computed RFs using the adjoint method (columns 1-2) and measurement method (columns 3-4) for a counter-clockwise circular-core spiral wave with Barkley kinetics BA1. The black line is the isoline $u=0.5$; the spiral wave tip is situated in the top-left quadrant. Results in columns $1-2$ were computed with dxspiral on a polar grid $N_{r}=120, R=12, N_{\theta}=128$. Results in columns 3-4 were found using the measurement method with $d x=0.1$, $N_{x}=N_{y}=240, d t=0.002375$, tip trajectory fitting for $t \in[40,80]$, tip calculation every 0.1 t.u., $A=0.05, k=1 /(2 d x), \lambda=10$.

than the time of $5-10 \mathrm{~min}$. that is needed using dxspiral. Nevertheless, by this comparison we show that the measurement method can be used to compute RFs in detail. The difference between both methods is displayed in Fig. 4.

\section{B. Flower-like meander with Barkley kinetics}

For the BA2 model parameters, the obtained RFs are shown in Figs. 5 and 6. Note that in a frame of reference with rotation frequency $\omega_{0}$, the RFs would be periodic in $\Psi$. 

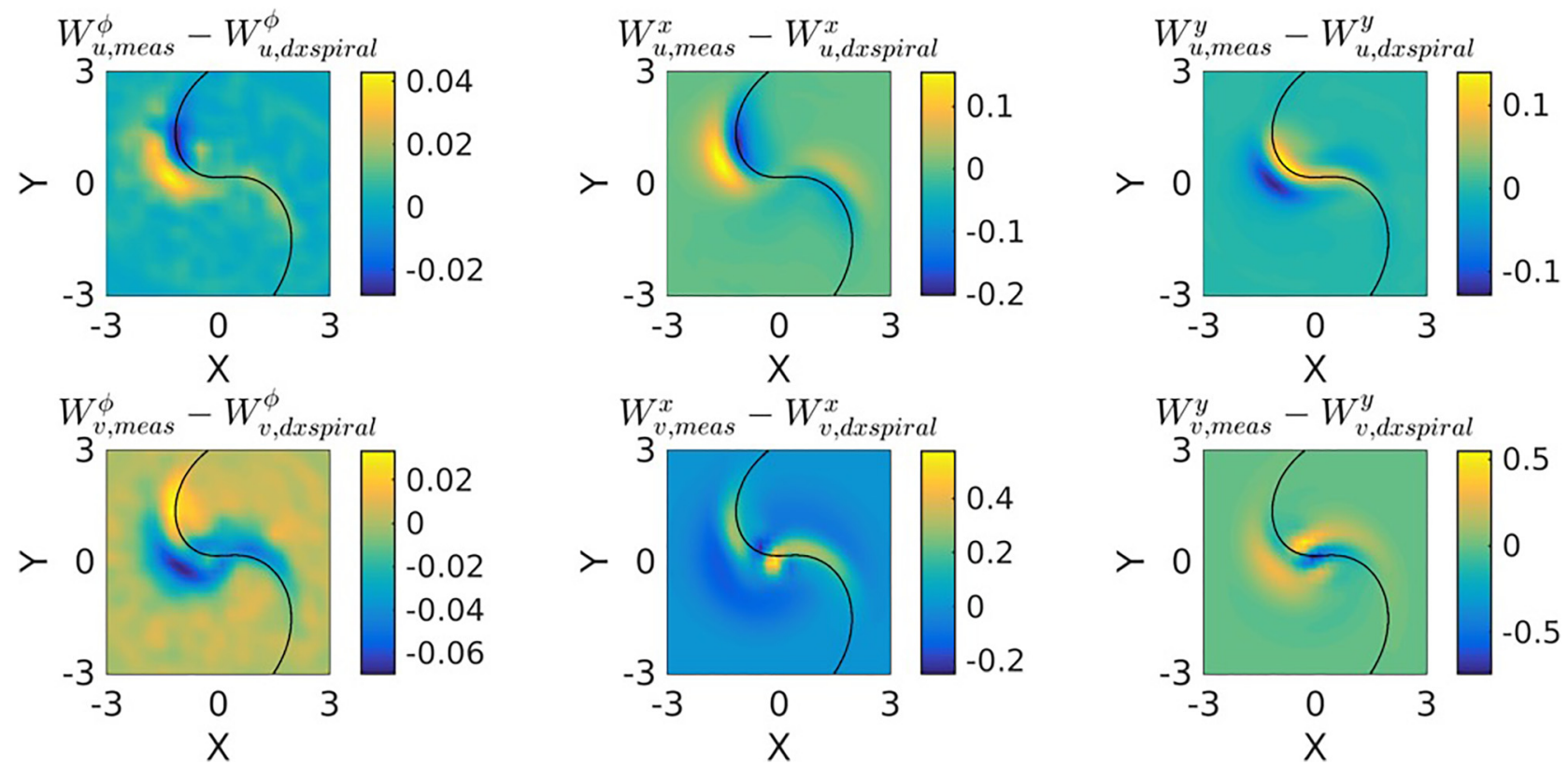

FIG. 4. Difference between the computed RFs using the adjoint method (dxspiral) and measurement method for the spiral wave from Fig. 3.
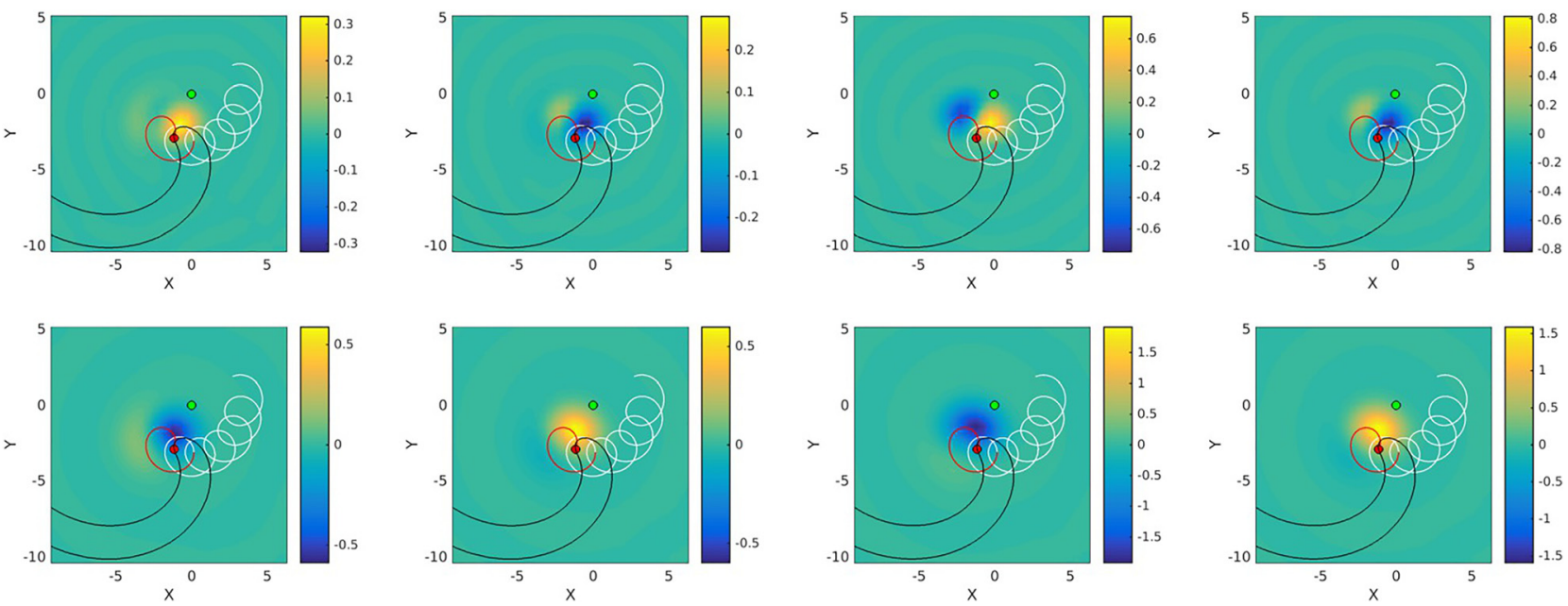

FIG. 5. Measured RFs for a meandering spiral wave with Barkley kinetics (BA2), with $d x=0.1, N_{x}=N_{y}=400, d t=0.002375$, tip trajectory fitting for $t \in[40,80]$, tip calculation every 0.1 t.u., $A=0.02, \sigma=2 d x, \lambda=500$. Results are shown for the meander phase corresponding to the spiral tip position at the red dot. The green square marks the meander center and the black line is the isoline $u=0.5$, showing the position of the wave front and wave back. The red trace is the tip trajectory during one meander cycle.
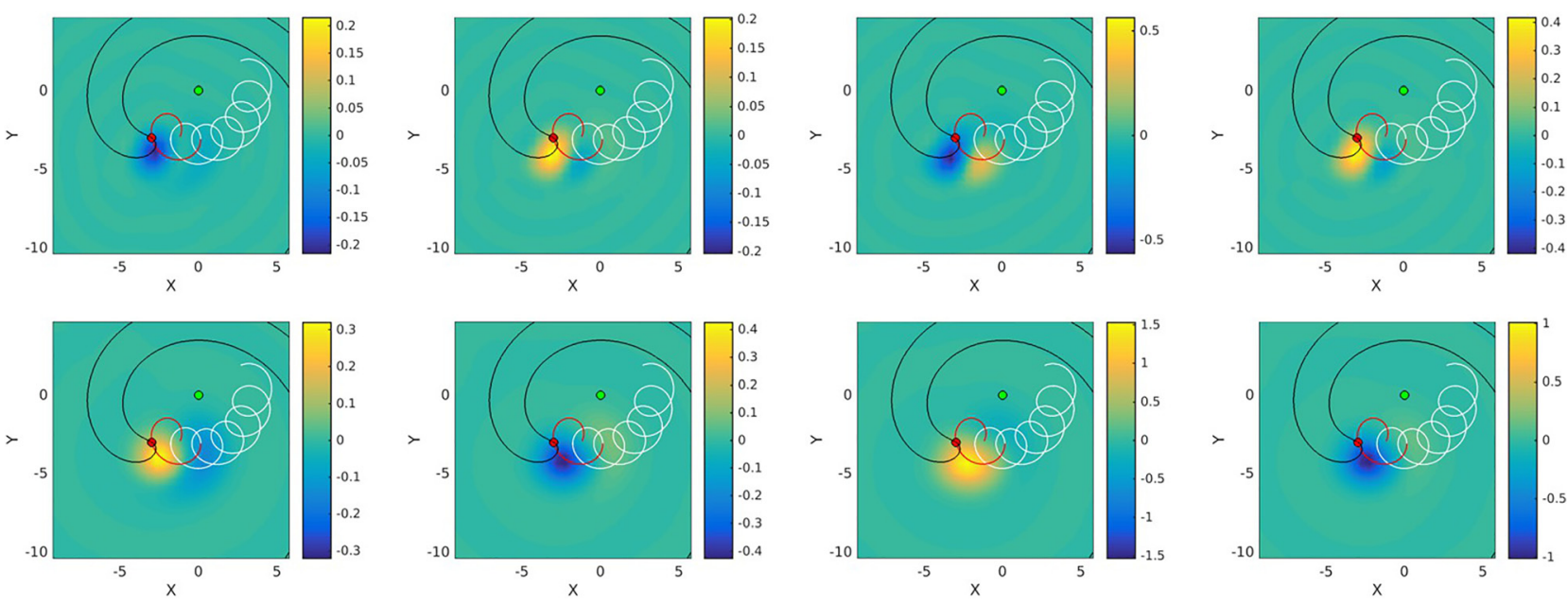

FIG. 6. Same as Fig. 5, at a different phase of the meander cycle, i.e., 2.5 t.u. or 0.5 meander periods later. 

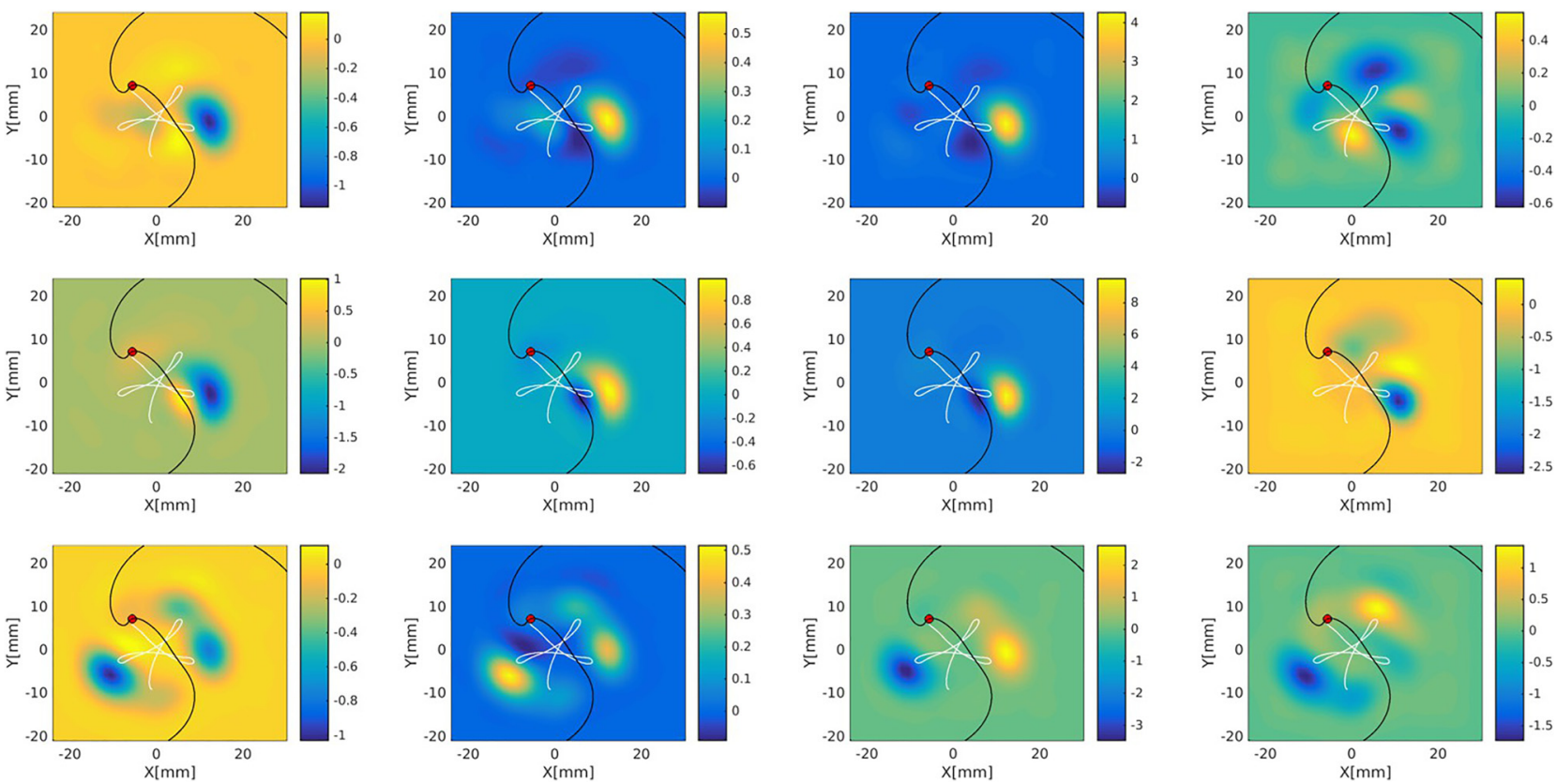

FIG. 7. Computed response functions for the FK cardiac tissue model, for a given meander phase denoted by spiral wave position (black line, $u=0.5)$ and tip position (red dot). The white trace is the upcoming unperturbed tip trajectory.

We show two cases of $\Psi$ in Figs. 5 and 6. Here too, the four RFs are strongly localized in the tip region.

\section{Linear-core meander with Fenton-Karma kinetics}

Applying the measurement procedure to the FK-GP model delivers the RFs shown in Figs. 7 and 8. For these two frames in the meander cycle, one can see that for all state variables, the RFs are localised near the turning points of the tip trajectory. If this holds for all times during the meander cycle, it is an important finding, since it means that external stimuli cannot change the rotor's path if they are not applied at locations where the few next turning points will occur. The FK model was created to represent fast and slow
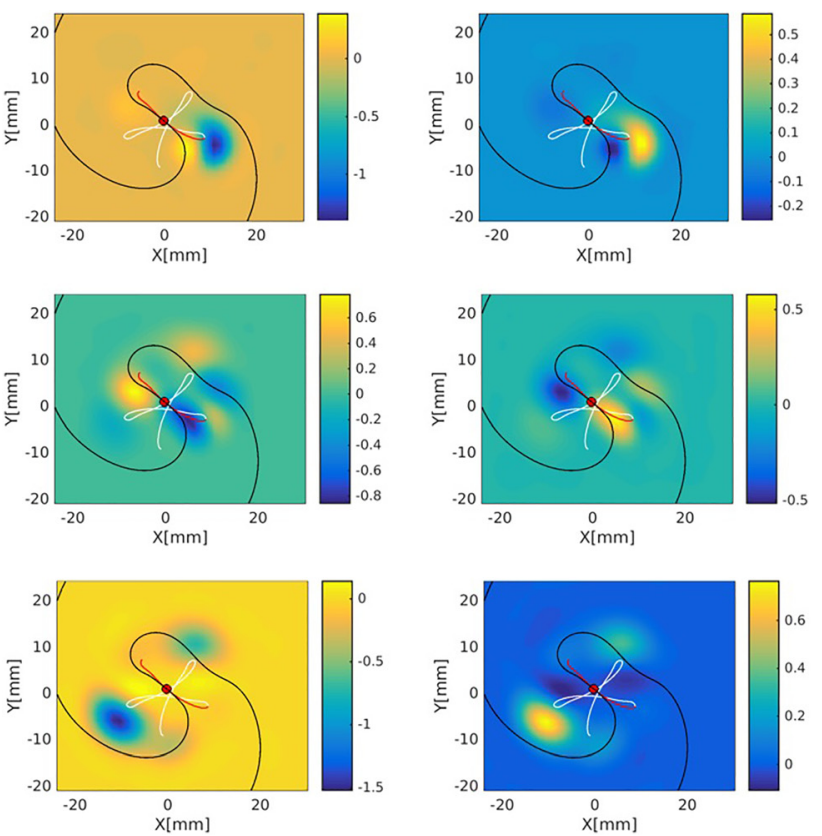

recovery processes by the $v$ and $w$ variables, respectively. From the RF structure, we note that the $\mathbf{W}_{v}^{\mu}$ are solely peaked at the next turning point, which can be understood since a stimulus in $v$ will have decayed when the second turning point is reached after $2 T_{0}=150 \mathrm{~ms}$. Contrarily, perturbing $w$ will affect significantly the spiral wave dynamics at the 4 upcoming points, as is evident from the $W_{w}^{\Psi}$ and $W_{w}^{\Phi}$ components. As expected, the effect on the phases $\Psi, \Phi$ has the same sign for all the turning points. This is not the case for the $W^{x}$ and $W^{y}$ components, since they depend on the orientation (i.e., have a tensor index which needs to be transformed). There, we also plot $\sqrt{\left(\mathbf{W}^{x}\right)^{2}+\left(\mathbf{W}^{y}\right)^{2}}$ for all 3 state variables in Fig. 9. For the rotational modes too, we note the
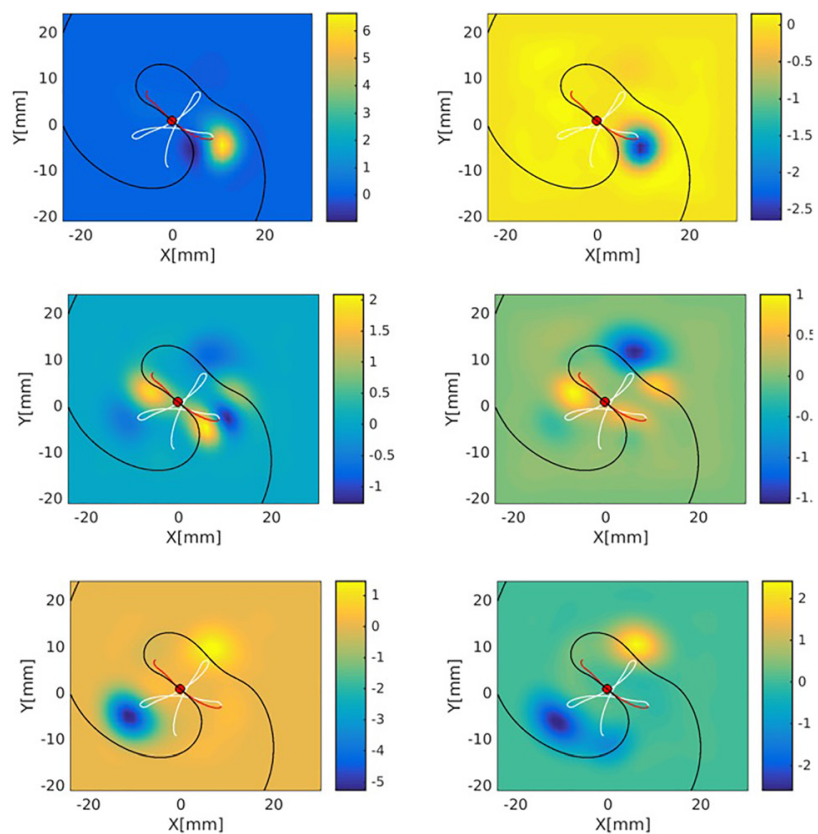

FIG. 8. Same as Fig. 7, at a different phase of the meander cycle, i.e., $37 \mathrm{~ms}$ or 0.5 meander periods later. 

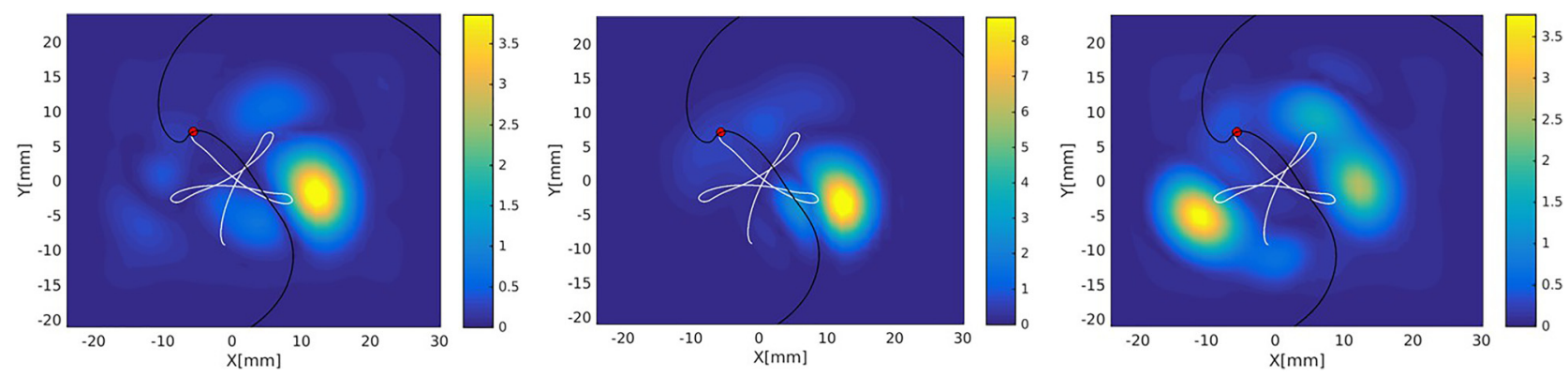

FIG. 9. Amplitude of the translational RF components, showing localisation near the upcoming turning points of the spiral wave tip trajectory.

memory effect is longest for the $w$ component. Moreover, the maximal amplitude is only found at the second turning point, probably since the tissue there is unexcited at the time of stimulus, contrary to the location of the first turning point.

\section{DISCUSSION}

We have developed and presented an "experimental" method to probe spiral wave response functions, and validated it on a numerical simulation of a circular-core spiral wave. In terms of numerical simulations, the method used is rather inefficient compared with other existing methods, but we nevertheless want to demonstrate three important points.

First, if one has sufficient computational power at one's disposal, it is possible to compute spiral wave RFs using minimal adaptation to the existing computer codes. By performing runs sequentially or on a cluster, there are no bigger memory limitations than for solving the original system.

Second, we see most use of the presented method in a truly experimental determination of spiral wave response functions, e.g., in the BZ-reaction or cardiac monolayers where Euclidean symmetry is more likely to be observed than in cardiac tissue. Note that in real-life experiments, the preparation only needs to be set up once. For, if the medium properties are sufficiently homogeneous (e.g., in BZ-reaction), one can perturb the spiral, let it rotate for 5-10 rotations to measure the shifts, then perturb again and so on. As a result, one may, e.g., infer 20 points of the RF from 100 spiral wave rotations. For cardiac tissue, pinning effects may be pronounced and therefore one may turn to monolayers of cultured tissue first.

Some future applications may lie outside the context of reaction-diffusion systems. The location and timing of the stimuli need not occur on a regular grid in time or space, enabling to take a Monte-Carlo approach, and better determine the RF at every new experiment in the series.

Third, by computing the RF for the FK-GP cardiac tissue model, we have demonstrated that the spiral wave RFs are concentrated near the turning points of the tip trajectory. Thus, the efforts to control or steer rotors in cardiac tissue, even if delivered to the entire tissue, are only effective near the turning points. Conversely, the interaction of a cardiac rotor with ambient structure (inhomogeneities, anisotropy, and tissue thickness) in quasi-2D and 3D tissue is also likely to be dominated by the structures it encounters at the position of its turning points.

\section{ACKNOWLEDGMENTS}

We thank V. N. Biktashev, I. V. Biktasheva, and C. Marcotte for helpful discussions, as well as the entire team that developed dxspiral and made it publicly available. H.D. was supported by FWO-Flanders during part of this work. This work was carried out using the STEVIN Supercomputer Infrastructure at Ghent University, funded by Ghent University, the Flemish Supercomputer Center (VSC), the Hercules Foundation and the Flemish Government department EWI.

${ }^{1}$ S. Nettesheim, A. von Oertzen, H. H. Rotermund, and G. Ertl, "Reaction diffusion patterns in the catalytic cooxidation on pt(110): Front propagation and spiral waves," J. Chem. Phys. 98, 9977-9985 (1993).

${ }^{2}$ W. Jahnke, C. Henze, and A. T. Winfree, "Chemical vortex dynamics in three-dimensional excitable media," Nature 336, 662-665 (1988).

${ }^{3}$ F. Siegert and C. J. Weijer, "Three dimensional scroll waves organize dictyostelium slugs," Proc. Natl. Acad. Sci. U. S. A. 89, 6433-6437 (1992).

${ }^{4}$ J. Lechleiter, S. Girard, E. Peraltal, and D. Clapham, "Spiral calcium wave propagation and annihilation in Xenopus Laevis oocytes," Science 252, 123-126 (1991).

${ }^{5}$ N. A. Gorelova and J. J. Bures, "Spiral waves of spreading depression in the isolated chicken retina," J. Neurobiol. 14, 353-363 (1983).

${ }^{6}$ A. M. Pertsov, J. M. Davidenko, R. Salomontsz, W. Baxter, and J. Jalife, "Spiral waves of excitation underlie reentrant activity in isolated cardiac muscle," Circ. Res. 72, 631-650 (1993).

${ }^{7}$ R. A. Gray, J. Jalife, A. Panfilov, W. T. Baxter, C. Cabo, and A. M. Pertsov, "Non-stationary vortex-like reentrant activity as a mechanism of polymorphic ventricular tachycardia in the isolated rabbit heart," Circulation 91, 2454-2469 (1995).

${ }^{8}$ R. H. Clayton, E. A. Zhuchkova, and A. V. Panfilov, "Phase singularities and filaments: Simplifying complexity in computational models of ventricular fibrillation," Prog. Biophys. Mol. Biol. 90, 378-398 (2006).

${ }^{9}$ S. M. Narayan, D. E. Krummen, K. Shivkumar, P. Clopton, W.-J. Rappel, and J. M. Miller, "Treatment of atrial fibrillation by the ablation of localized sources," J. Am. Coll. Cardiol. 60(7), 628-636 (2012).

${ }^{10}$ R. A. Gray, A. M. Pertsov, and J. Jalife, "Spatial and temporal organization during cardiac fibrillation," Nature 392, 75-78 (1998).

${ }^{11}$ F. H. Fenton and A. Karma, "Vortex dynamics in three-dimensional continuous myocardium with fiber rotation: Filament instability and fibrillation," Chaos 8, 20-47 (1998).

${ }^{12}$ I. V. Biktasheva and V. N. Biktashev, "Wave-particle dualism of spiral wave dynamics,” Phys. Rev. E 67, 026221 (2003).

${ }^{13}$ J. P. Keener, "The dynamics of three-dimensional scroll waves in excitable media," Physica D 31, 269-276 (1988).

${ }^{14}$ V. N. Biktashev, A. V. Holden, and H. Zhang, "Tension of organizing filaments of scroll waves," Philos. Trans. R. Soc. London, A 347, 611-630 (1994).

${ }^{15}$ H. Verschelde, H. Dierckx, and O. Bernus, "Covariant stringlike dynamics of scroll wave filaments in anisotropic cardiac tissue," Phys. Rev. Lett. 99, 168104 (2007).

${ }^{16} \mathrm{H}$. Dierckx and H. Verschelde, "Effective dynamics of twisted and curved scroll waves using virtual filaments," Phys. Rev. E 88, 062907 (Dec 2013). 
${ }^{17} \mathrm{H}$. Henry, "Spiral wave drift in an electrical field and scroll wave instabilities," Phys. Rev. E 70, 026204 (2004).

${ }^{18}$ H. Dierckx, E. Brisard, H. Verschelde, and A. V. Panfilov, "Drift laws for spiral waves on curved anisotropic surfaces," Phys. Rev. E 88(1), 012908 (2013).

${ }^{19}$ H. Dierckx, S. Arens, B.-W. Li, L. D. Weise, and A. V. Panfilov, "A theory for spiral wave drift in reaction-diffusion-mechanics systems," New J. Phys. 17(4), 043055 (April 2015).

${ }^{20} \mathrm{~K}$. Kuramoto, "Instability and turbulence of wave fronts in reactiondiffusion systems," Prog. Theor. Phys. 63, 1885-1903 (1980).

${ }^{21} \mathrm{H}$. Dierckx, O. Bernus, and H. Verschelde, "Accurate eikonal-curvature relation for wave fronts in locally anisotropic reaction-diffusion systems," Phys. Rev. Lett. 107, 108101 (2011).

${ }^{22}$ J. Löber and H. Engel, "Controlling the position of traveling waves in reaction-diffusion systems," Phys. Rev. Lett. 112(14), 148305 (2014).

${ }^{23} \mathrm{~V}$. N. Biktashev and A. V. Holden, "Resonant drift of autowave vortices in two dimensions and the effect of boundaries and inhomogeneities," Chaos, Solitons Fractals 5, 575-662 (1995).

${ }^{24}$ C. D. Marcotte and R. O. Grigoriev, "Adjoint eigenfunctions of temporally recurrent single-spiral solutions in a simple model of atrial fibrillation," Chaos 26(9), 093107 (2016).

${ }^{25}$ H. Dierckx, I. V. Biktasheva, H. Verschelde, A. V. Panfilov, and V. N. Biktashev, "Filament tension and phase-locking of meandering scroll waves," Phys. Rev. Lett. (to be published).
${ }^{26}$ I. V. Biktasheva, Y. E. Elkin, and V. N. Biktashev, "Localized sensitivity of spiral waves in the complex Ginzburg-Landau equation," Phys. Rev. E 57, 2656-2659 (1998).

${ }^{27} \mathrm{H}$. Henry and V. Hakim, "Scroll waves in isotropic excitable media: Linear instabilities, bifurcations, and restabilized states," Phys. Rev. E 65, 046235 (2002).

${ }^{28}$ I. V. Biktasheva, D. Barkley, V. N. Biktashev, G. V. Bordyuogov, and A. J. Foulkes, "Computation of the response functions of spiral waves in active media," Phys. Rev. E 79, 056702 (2009).

${ }^{29}$ V. N. Biktashev, I. V. Biktasheva, and N. A. Sarvazyan, "Evolution of spiral and scroll waves of excitation in a mathematical model of Ischaemic border zone," PLoS One 6(9), e24388 (2011).

${ }^{30}$ C. D. Marcotte and R. O. Grigoriev, "Unstable spiral waves and local Euclidean symmetry in a model of cardiac tissue," Chaos 25(6), 063116 (2015).

${ }^{31}$ A. N. Tikhonov, "Solution of incorrectly formulated problems and regularization method," Doklady Akad. Nauk SSSR 151(3), 501 (1963).

${ }^{32}$ MATLAB 6.1, The MathWorks Inc., Natick, MA, 2000, see www.mathworks.com.

${ }^{33}$ D. Barkley, "A model for fast computer simulation of waves in excitable media," Physica D 49, 61-70 (1991).

${ }^{34}$ W. Gander, G. H. Golub, and R. Strebel, "Least-squares fitting of circles and ellipses," BIT 34, 558-578 (1994).

${ }^{35}$ D. Barkley, V. N. Biktashev, I. V. Biktasheva, G. V. Bordyugov, and A. J. Foulkes, http://cgi.csc.liv.ac.uk/ivb/SOFTware/DXSpiral.html for Dxspiral: A code for studying spiral waves on a disk (2010). 